\title{
The Role of Immune Escape and Immune Cell Infiltration in Breast Cancer
}

\author{
André Steven Barbara Seliger \\ Institute of Medical Immunology, Martin Luther University Halle-Wittenberg, Halle (Saale), Germany
}

\section{Keywords}

Breast cancer - Immune escape - Immune surveillance . Tumor microenvironment · Immune response

\section{Summary}

While detailed analysis of aberrant cancer cell signaling pathways and changes in cancer cell DNA has dominated the field of breast cancer biology for years, there now exists increasing evidence that the tumor microenvironment (TME) including tumor-infiltrating immune cells support the growth and development of breast cancer and further facilitate invasion and metastasis formation as well as sensitivity to drug therapy. Furthermore, breast cancer cells have developed different strategies to escape surveillance from the adaptive and innate immune system. These include loss of expression of immunostimulatory molecules, gain of expression of immunoinhibitory molecules such as PD-L1 and HLA-G, and altered expression of components involved in apoptosis. Furthermore, the composition of the TME plays a key role in breast cancer development and treatment response. In this review we will focus on i) the different immune evasion mechanisms used by breast cancer cells, ii) the role of immune cell infiltration in this disease, and (iii) implication for breast cancer-based immunotherapies.

\section{Introduction}

There is evidence that the low immunogenicity and the intense immunosuppressive tumor microenvironment (TME) of breast cancers (BC) limit the benefit of immunotherapies targeting the adaptive immune system, such as checkpoint inhibitors (CPI) [1, 2]. Immunosuppressive mechanisms are essential for the development of normal mammary glands; these strategies are also used by $\mathrm{BC}$ cells to promote tumor tolerance and escape from immune surveillance in the early stages of disease, suggesting an important role of adaptive and innate immunity in $\mathrm{BC}$ development and progression. Increased insights into the molecular mechanisms employed by cancer cells to subvert or escape from immune recognition have recently opened an array of new therapeutic interventions. These include the implementation of monoclonal antibodies directed against neoantigens, the development of cancer vaccines, adoptive T-cell transfer, and the use of immunomodulatory agents such as cytokines, costimulatory receptor agonists, and CPI. Therefore, we here review the different immune escape strategies of $\mathrm{BC}$ as well as the role of tumor-infiltrating immune cells in the TME and their clinical relevance, finally suggesting the development of novel strategies to overcome immunosuppression and to enhance the immunogenicity of tumors by reverting their immune escape with the aim to develop and/or improve immunotherapies for this malignancy.

\section{Immune Escape Mechanisms of Tumors}

Recent evidence has demonstrated an essential role of cells from the innate and adoptive immune system involved in the initiation but also progression of cancer. This is mediated by the suppression of immune rejection leading to enhanced tumor growth and spread including the formation of the primary metastatic lesions [3, 4]. In the early steps of tumor development, host immune factors, in par-

\section{KARGER \\ Fax +497614520714

() 2018 S. Karger GmbH, Freiburg
Prof. Dr. Barbara Seliger 
ticular cells of the innate immune system such as natural killer (NK) cells, tumor-associated macrophages, tumor-associated neutrophils, and myeloid-derived suppressor cells, play a key role in the rejection of cancer cells [5], while in the equilibrium phase tumor cells survive in a quiescent state [6]. This is followed by an immune escape in which tumor variants grow out and evade immune recognition and establish an immunosuppressive TME [7, $8]$. This so-called immunoediting process suggests that tumors develop distinct mechanisms to evade immune surveillance, induce tolerance, and survive in the host. Only recently, immune evasion has also been recognized as a hallmark of $\mathrm{BC}$ [9]. The underlying mechanisms include reduced expression of major histocompatibility complex (MHC) class I, adhesion and costimulatory molecules, loss of antigens, and increased expression of immunosuppressive components such as HLA-G, HLA-E, and PD-L1 and of other immunosuppressive factors such as cytokine and metabolites that contribute to the escape from immune recognition [10]. Due to these alterations and the influence of host immunity, tumors develop low immunogenicity and a strong immunosuppressive TME and do not elicit an adaptive immune response. Furthermore, the frequency of CD4+ T cells and CD8+ cytotoxic T lymphocytes (CTL) in the TME is low, and their phenotype is often associated with immune exhaustion [11]. Based on this information, a number of studies are currently ongoing analyzing the nature of immune cells in the TME and the peripheral blood of tumor patients in combination with the cytokine profile produced by these cells and the immunogenic phenotype of tumor cells.

\section{Breast Cancer and Its Immunogenicity}

$\mathrm{BC}$ has been suggested to be a very heterogeneous disease, and based on histology and the molecular and transcriptional profile, different BC subtypes have been identified and correlated with clinical outcome. Primarily, BC have been classified based on the presence or absence of certain hormone receptors, growth factor receptors, and mutational load resulting to the definition of different BC subtypes. Recently, a link between their molecular make up and immunologic features was reported based on RNA sequencing data of the The Cancer Genome Atlas (TCGA) [12].

\section{Breast Cancer and Tumor-Associated Antigens}

High throughput technologies allowed the identification of structural alterations, such as gene mutations, amplifications, and loss of heterozygosity $(\mathrm{LOH})$, in $\mathrm{BC}$ subtypes. This can result in the development of neoantigens presented by human leukocyte antigen (HLA) class I molecules, which can then be recognized as foreign by CD8+ CTL. Next to these mutation-specific neoantigens, other tumor-associated antigens (TAA) are autoantigens which are expressed excessively, differentiation-specific antigens, cancer testis genes (CTA) that display normal expression in immune-privileged organs but aberrant expression in several types of cancer in- cluding BC-modified autoantigens, TAA that are highly expressed in tumors such as HER2/neu, and viral-associated antigens. For example, members of the CTA family of melanoma-associated antigens (MAGEs), MAGE-A9 and MAGE-A11, are less expressed in estrogen receptor(ER)-negative and HER2/neu-negative BC [13, 14]. In contrast, upregulation of MAGE-A9 in invasive ductal BC is correlated with an unfavorable outcome for patients [15]. Furthermore, mesothelin is overexpressed in triple-negative breast cancer (TNBC) via modifying the T-cell receptor (TCR) [16]. Other TAA expressed by BC cells include MUC1, Claudin, and HER2/neu [17, 18]. The latter is highly overexpressed in HER2/neu-positive BC, and its expression levels are positively correlated with histologic tumor grade and associated with poor prognosis [19-22]. Although low expression of HER2/neu might restrict the immune cell-mediated destruction of tumor cells, high levels of HER2/neu have been shown to diminish HLA class I surface expression resulting in reduced CTL activity [23]. Loss or modification of surface antigens may promote immune evasion via a lack of tumor cell recognition. These distinct TAA and mechanisms may become potential targets to broaden the immunotherapeutic strategies in BC.

\section{Immunogenicity of Breast Cancer and Immune Escape}

Recent data suggest that $\mathrm{BC}$ might also be an immunogenic tumor. Using RNA sequencing data from the TCGA obtained from $>1,004$ BC cases, 4 distinct immune phenotypes were identified based on the expression of immune-relevant genes. These Immunologic Constant of Rejection (ICR) phenotypes, ICR1-4, define an immune-favorable phenotype (ICR4) and immune-unfavorable phenotypes (ICR1) associated with tumor progression and survival of $\mathrm{BC}$ patients [12].

\section{Abnormal Expression of MHC Class I and Components of the Antigen Processing and Presentation Machinery and Interferon} Signal Transduction Pathway in BC

The MHC class I/TAA complex expressed on tumor cells is recognized by CD8+ effector CTL. Loss or downregulation of MHC class I surface antigens allows tumor cells to escape immune surveillance $[24,25]$. Tumor cells of distinct origin are able to silence MHC class I surface expression, which is often caused by diminished expression of components of the MHC class I antigen processing and presentation machinery (APM) and of the interferon (IFN) signal transduction pathway and associated with a worse outcome [26]. Concerning BC, HLA class I expression levels have been shown to be significantly downregulated in $\mathrm{BC}$, which is necessary for the transformation of normal cells into abnormal cells. Furthermore, downregulation of beta- 2 microglobulin $\left(\beta_{2}-\mathrm{m}\right)$, calnexin, and transporter-associated antigen processing (TAP) subunit 1 leading to impaired HLA class I expression was found in metastatic brain lesions of $\mathrm{BC}$, which was also negatively linked to the frequency of CTL infiltration [27]. The underlying molecular mechanisms responsible for deficient HLA class I expression are diverse and could be attributed to gene mutations, $\mathrm{LOH}$, and tran- 
scriptional, posttranscriptional, and translational control, and altered oncogenic and IFN signaling [28-33]. This results in decreased tumor immunogenicity, which is consequently associated with increased malignancy, metastasis formation, worse prognosis, and poorer response to (immuno-)therapies [34-37]. While downregulation of HLA class I antigens negatively interferes with T-cell responses only, the expression of the non-classical HLA-G, which consists of 4 membrane-bound and 3 soluble isoforms [38], results in escape of tumor cells from both NK- and T-cell-mediated recognition [39]. In BC, increased HLA-G expression levels were found [40] which were not only associated with poor prognosis $[41,42]$ but also with therapy response of $\mathrm{BC}$ treated with neoadjuvant chemotherapy [40]. In addition, high soluble HLA-G concentrations were detected in the serum of $\mathrm{BC}$ patients and correlated with occurrence of metastasis, suggesting their usefulness as prognostic biomarker [43]. However, whether HLA-G could be detected in exosomes and thus spread to other cells to reduce their immunogenic phenotype remains controversial [44]. The role of HLA-G in BC was further underlined by the fact that the $14 \mathrm{bp}$ InDel polymorphism in the HLA-G gene was a risk factor for the development of BC [45]. Similar to HLA-G, the stress-induced MHC class I-related gene $\mathrm{A}(\mathrm{MICA})$ is also frequently upregulated in high-grade $\mathrm{BC}$ and is an indicator of poor prognosis [46]. Taken together, the link between low HLA class I/high HLA-G/MICA expression and worse $\mathrm{BC}$ prognosis postulates the induction of HLA class I expression and inhibition of HLA-G/MICA expression as a new therapeutic option in the treatment of $\mathrm{BC}$ [47].

\section{Disturbance of Antiapoptotic Function in Breast Cancer}

Next to alterations of classical and non-classical HLA class I genes, reduced apoptosis represents a major strategy for evading immune response in cancer development [48]. This includes altered expression of Fas (factor associated with suicide), also known as CD95, and its ligand FasL (CD95L) [49]. This system can activate apoptosis signaling and induce apoptosis in cells. Increased FasL levels in BC cells cause effector T lymphocytes to die, resulting in escape from immune surveillance [50, 51]. Furthermore, tumor cells can resist Fas-mediated apoptosis by silencing or downregulating the Fas/FasL signaling pathways, which has been reported to be associated with a worse prognosis in BC. It is noteworthy that CD95L exists not only at the transmembrane but also in a soluble form (sFasL) due to cleavage by metalloproteases. sFasL has been found in the serum of TBNC patients promoting metastatic dissemination [52]. In addition to Fas/FasL, other proteins such as Bcl-2, survivin, and caspase play an important role in apoptosis in $\mathrm{BC}$. Bcl-2 is overexpressed in $\mathrm{BC}$ cells leading to prevention of apoptosis associated with neoplastic transformation and an enhanced cellular live span $[53,54]$. Expression of survivin, another member of the antiapoptotic family, is increased in BC. This is accompanied by poor outcome, advanced tumor grade, increased metastasis formation, and a low survival rate of patients [55]. In contrast, decreased caspase activation due to downregulation of caspase expression represents a further strategy of tumors to resist apoptosis. In this context, it is noteworthy that caspase- 3 and -7 expression is downregulated in BC [56], but this deregulation appear not to be significantly correlated with the clinicopathologic features of this disease [57].

\section{Expression of Co-Inhibitory Molecules}

Based on the successful implementation of antibodies directed against immune checkpoints, the programmed death receptor 1 (PD-1) and its ligand PD-L1 have come into the focus of research $[58,59]$. The interaction between PD-1 on T lymphocytes with PD-L1 expressed on the surface of tumor cells inhibits the activation of effector T cells and induces FasL and the immunosuppressive cytokine interleukin (IL)-10. PD-L1 is expressed in many tumor cells of distinct origin and at a high frequency on $\mathrm{BC}$ cells [60] and circulating tumor cells [61]. Inhibition of PD-L1 significantly blocks T-cell apoptosis in tumor models [61, 62]. PD-L1 could directly synergize with FOXP3+ regulatory T cells [62], but could also be affected by ubiquitination and N-glycosylation [63]. In different $\mathrm{BC}$ subtypes, heterogeneous expression of PD-1/PD-L1 was shown [64-66]. In particular in TNBC, PD-L1 was found to be overexpressed $[67,68]$, which is related to tumor grade $[69,70]$, local cytotoxic immune response, and prognosis [71]. This was the rationale for the implementation of anti-PD1/anti-PD-L1 for the treatment of TNBC, stimulating tumor regression and improving patient outcomes. Indeed, results from clinical trials demonstrated an increase in lasting local antitumor responses accompanied by a promising clinical benefit $[72,73]$. Other co-inhibitory molecules include the lymphocyte activation gene 3 (LAG-3) and the T cell immunoglobulin and mucin domain-3 (TIM-3) checkpoints, which have recently been investigated in a large cohort of BC patients and their expression pattern linked to clinicopathologic parameters. LAG-3-positive tumor-infiltrating lymphocytes (TILs) were particularly enriched in ER-negative BC and were negatively associated with young age, large tumor size, and high proliferation, while a high proportion of PD-1/PD-L1-positive tumors were coinfiltrated with LAG-3-positive TILs [74]; TIM-3 expression levels were high on tumor cells and significantly associated with clinicopathologic parameters such as age, axillary lymph node metastasis, and TNM stage [75]. In addition, other B7 family members, such as B7-H3 known to stimulate IL-10 secretion, also contribute to tumor immune evasion and tumor progression. Expression of B7-H3 was found at a high frequency of BC lesions, which was correlated with IL-10 [76]. Thus, other immune checkpoints also promote $\mathrm{BC}$ development and progression and might be used as independent prognostic factors for invasive ductal carcinoma (IDC) patients.

\section{Tumor Microenvironment in Breast Cancer}

Different components of the TME have been show to play a key role in tumor development and progression. These are cellular, soluble, and physical factors which are shaped by specific structures, functions, and metabolic properties of neoplastic lesions [77, 78]. Autocrine and paracrine mechanisms of tumor cells alter the inter- 
play between tumor and immune cells thereby maintaining conditions essential for the survival, development, proliferation, and progression of tumors. Furthermore, advances in molecular biologic as well as immunologic features of $\mathrm{BC}$ have helped elucidate the association between malignant $\mathrm{BC}$ cells and immune factors or their modulators around the tumor. Different types of immune responses have been linked to a distinct cytokine microenvironment: While tumor-specific B-cell responses were correlated with increased transforming growth factor (TGF)- $\beta 1$, reduced IFN- $\alpha$, absence of T-cell responses, and worse prognosis, high IFN- $\gamma$ levels were associated with a strong presence of T-cell infiltration and activity [79]. Recently, a number of studies characterized the BC TME concerning suppressive immune cells, re-programmed fibroblast cells, altered extracellular matrix, and soluble factors, which negatively interfere with an effective antitumor response and promote BC progression and metastasis. It has been suggested that changes in the TME not only have an important impact on BC development and progression but appear to also serve as prognostic factors for the clinical outcome of patients and response to (immuno-)therapies. Substantive tissue and tumor subtype-specific differences of multiple cell types, in particular TILs, have been identified in BC subtypes, and in particular TNBC and HER2/neu-negative BC exhibit a unique TME distinct from that of other BC subtypes. Immunohistochemical analyses have demonstrated that CD8+ CTL, known to contribute to tumor clearance, were associated with good prognosis and long-term survival [80] and are thus of clinical relevance [81]. Furthermore, the number of tumor-infiltrating CD8+ T cells was associated with primary tumor size, lymph node metastasis, WHO (World Health Organization) grade, Ki-67, and molecular classification. Although the major focus of most studies was on CD8+ CTL, Th1 CD4+ T cells could also contribute to the elimination of $\mathrm{BC}$ by the production of IFN- $\gamma$, resulting in reduced angiogenesis and enhanced T-cell and M1 macrophage activity [82]. In contrast, the role of Th2 CD4+ T cells has not yet been identified in detail in $\mathrm{BC}$, but might be more tumor-promoting than tumor-suppressive. There is also evidence that infiltration with CXCL13-expressing CD4+ follicular helper cells in BC predicts improved patient survival [83] and could serve as a prognostic marker in this disease [84]. The function of B-cell infiltration is also poorly understood, and discrepant results have been reported regarding its importance, or lack thereof, for poor/good clinical prognosis [85]. Therefore, more detailed analyses have to be performed in order to conclusively determine the prognostic and predictive potential of immune cells in BC. Expression profiling of BC lesions further revealed a differential expression pattern of genes associated with immune cells, such as IFN-regulated genes [86, 87], B-lymphocyte marker [86], as well as T-lymphocyte-associated genes [87], which further underpins the crucial role even of host immune responses in this disease. This was also underlined and extended by a recent report comparing the expression of immune-relevant genes in normal mammary tissues, ductal carcinoma in situ (DCIS), and IDC [88]. A decrease in CD8+ signatures in IDCs versus DCIS and of activated $\mathrm{GZMB}+\mathrm{CD} 8+\mathrm{T}$ cells in IDC was detected. These were accompanied by significantly higher TCR clonotype diversity in DCIS than in IDCs. Furthermore, a link between the frequency of TILs in the stroma and prognosis as well as response to chemotherapy has been shown [89-91].

\section{Perspectives and Conclusion}

The main hurdle for $\mathrm{BC}$ in generating a broad and robust antitumor immune response is to overcome the evasion of immune surveillance. However, the underlying molecular mechanisms leading to immune escape in $\mathrm{BC}$ have to still be elucidated in detail. Despite some treatment success with CPIs, the response seen in patients is limited. The cause of intrinsic and extrinsic resistance mechanisms of $\mathrm{BC}$ have not yet been analyzed. However, first evidence in melanoma patients suggests that resistance to anti-PD1 therapy is mediated by structural alterations or reduced expression of HLA class I APM and/or IFN signaling components [92]. Future perspectives to improve patient outcome and monitor therapy response are therefore based on the molecular make up in combination with the expression of immune-relevant molecules in both immune cells and tumor cells and the thorough analysis of the composition of the immune cell infiltrate as well as the peripheral blood of BC patients. This might lead to the development and design of therapies in which CPIs will be combined with each other or with targeted therapies, chemotherapy, and/or radiation. Examples are anti-PD-1/anti-PD-L1 or inhibitors of MAP2K and glucocorticoid-induced tumor necrosis factor receptor (GITR) [93, 94], which are currently under way and will be covered by a separate article in this issue.

\section{Disclosure Statement}

We hereby state that both authors have no conflicts of interest.

\section{References}

1 Rugo HS, Delord J-P, Im S-A, Tan A: Preliminary efficacy and safety of pembrolizumab (MK-3475) in patients with PD-L1-positive, estrogen receptor-positive $(\mathrm{ER}+) / \mathrm{HER} 2$ negative advanced breast cancer enrolled in KEYNOTE-028. San Antonio Breast Cancer Symposium 2015; abstr S5-07.

2 Solinas C, Gombos A, Latifyan S, et al: Targeting immune checkpoints in breast cancer: an update of early results. ESMO Open 2017;2:e000255.
3 Mittal D, Gubin MM, Schreiber RD, Smyth MJ: New insights into cancer immunoediting and its three component phases - elimination, equilibrium and escape. Curr Opin Immunol 2014;27:16-25.

4 Smyth MJ, Dunn GP, Schreiber RD: Cancer immunosurveillance and immunoediting: the roles of immunity in suppressing tumor development and shaping tumor immunogenicity. Adv Immunol 2006;90:1-50.
5 Spranger S, Sivan A, Corrales L, Gajewski TF: Tumor and host factors controlling antitumor immunity and efficacy of cancer immunotherapy. Adv Immunol 2016;130:75-93.

6 Vesely MD, Kershaw MH, Schreiber RD, Smyth MJ: Natural innate and adaptive immunity to cancer. Annu Rev Immunol 2011;29:235-271. 
7 Dunn GP, Bruce AT, Ikeda H, Old LJ, Schreiber RD: Cancer immunoediting: from immunosurveillance to tumor escape. Nat Immunol 2002;3:991-998.

8 Schreiber RD, Old LJ, Smyth MJ: Cancer immunoediting: integrating immunity's roles in cancer suppression and promotion. Science 2011;331:1565-1570.

9 Hanahan D, Weinberg RA: Hallmarks of cancer: the next generation. Cell 2011;144:646-674.

10 Fridman WH, Zitvogel L, Sautès-Fridman C, Kroemer $\mathrm{G}$ : The immune contexture in cancer prognosis and treatment. Nat Rev Clin Oncol 2017;14:717-734.

11 Dushyanthen S, Beavis PA, Savas P, et al: Relevance of tumor-infiltrating lymphocytes in breast cancer. BMC Med 2015;13:202.

12 Hendrickx W, Simeone I, Anjum S, et al: Identification of genetic determinants of breast cancer immune phenotypes by integrative genome-scale analysis. Oncoimmunology 2017;6:e1253654.

13 Abd-Elsalam EA, Ismaeil NA: Melanoma-associated antigen genes: a new trend to predict the prognosis of breast cancer patients. Med Oncol 2014;31:285.

14 Hou SY, Sang MX, Geng CZ, et al: Expressions of MAGE-A9 and MAGE-A11 in breast cancer and their expression mechanism. Arch Med Res 2014;45:44-51.

$15 \mathrm{Xu}$ X, Tang X, Lu M, et al: Overexpression of MAGEA9 predicts unfavorable outcome in breast cancer. Exp Mol Pathol 2014;97:579-584.

16 Tchou J, Wang LC, Selven B, et al: Mesothelin, a novel immunotherapy target for triple negative breast cancer. Breast Cancer Res Treat 2012;133:799-804.

17 Zieglschmid V, Hollmann C, Gutierrez B, et al: Heterogeneous expression of tumor-associated genes in disseminated breast cancer cells. Anticancer Res 2007;27: 1769-1776.

18 Mukherjee P, Tinder TL, Basu GD, et al: Therapeutic efficacy of MUC1-specific cytotoxic T lymphocytes and CD137 co-stimulation in a spontaneous breast cancer model. Breast Dis 2004;20:53-63.

19 Nicolini A, Ferrari P, Duffy MJ: Prognostic and predictive biomarkers in breast cancer: past, present and future. Semin Cancer Biol 2017;pii:S1044-579X(17) 30052-4.

20 Yan J, Liu XL, Han LZ, et al: Relation between Ki-67, ER, PR, Her2/neu, p21, EGFR, and TOP II- $a$ expression in invasive ductal breast cancer patients and correlations with prognosis. Asian Pac J Cancer Prev 2015;16:823-829.

21 Shokouh TZ, Ezatollah A, Barand P: Interrelationships between Ki67, HER2/neu, p53, ER, and PR status and their associations with tumor grade and lymph node involvement in breast carcinoma subtypes: retrospective-observational analytical study. Medicine (Baltimore) 2015;94:e1359.

22 Hoff ER, Tubbs RR, Myles JL, Procop GW: HER2/neu amplification in breast cancer: stratification by tumor type and grade. Am J Clin Pathol 2002;117:916-921.

23 Herrmann F, Lehr HA, Drexler I, et al: HER-2/neumediated regulation of components of the MHC class I antigen-processing pathway. Cancer Res 2004;64:215220.

24 Garrido F, Algarra I, García-Lora AM: The escape of cancer from T lymphocytes: immunoselection of MHC class I loss variants harboring structural-irreversible 'hard' lesions. Cancer Immunol Immunother 2010;59: 1601-1606.

25 Seliger B, Jasinski-Bergner S, Quandt D, et al: HLA-E expression and its clinical relevance in human renal cell carcinoma. Oncotarget 2016;7:67360-67372.

26 Respa A, Bukur J, Ferrone S, et al: Association of IFNgamma signal transduction defects with impaired HLA class I antigen processing in melanoma cell lines. Clin Cancer Res 2011;17:2668-2678.
27 Liu Y, Komohara Y, Domenick N, et al: Expression of antigen processing and presenting molecules in brain metastasis of breast cancer. Cancer Immunol Immunother 2012;61:789-801.

28 Carbotti G, Nikpoor AR, Vacca P, et al: IL-27 mediates HLA class I up-regulation, which can be inhibited by the IL-6 pathway, in HLA-deficient small cell lung cancer cells. J Exp Clin Cancer Res 2017;36:140.

29 Hanalioglu D, Ayvaz DC, Ozgur TT, et al: A novel mutation in TAP1 gene leading to MHC class I deficiency: report of two cases and review of the literature. Clin Immunol 2017;178:74-78.

30 Wang M, Zhang C, Song Y, et al: Mechanism of immune evasion in breast cancer. Onco Targets Ther 2017;10:1561-1573.

31 Zimmer J, Andrès E, Donato L, et al: Clinical and immunological aspects of HLA class I deficiency. QJM 2005;98:719-727.

32 Ryschich E, Cebotari O, Fabian OV, et al: Loss of heterozygosity in the HLA class I region in human pancreatic cancer. Tissue Antigens 2004;64:696-702.

33 Eichmüller SB, Osen W, Mandelboim O, Seliger B: Immune modulatory microRNAs involved in tumor attack and tumor immune escape. J Natl Cancer Inst 2017;109.

34 Mariya T, Hirohashi Y, Torigoe T, et al: Prognostic impact of human leukocyte antigen class I expression and association of platinum resistance with immunologic profiles in epithelial ovarian cancer. Cancer Immunol Res 2014;2:1220-1229.

35 Jobim MR, Jobim M, Salim PH, et al: Analysis of KIR gene frequencies and HLA class I genotypes in breast cancer and control group. Hum Immunol 2013;74: $1130-1133$.

36 Kaneko K, Ishigami S, Kijima Y, et al: Clinical implication of HLA class I expression in breast cancer. BMC Cancer 2011;11:454.

37 Garrido F, Cabrera T, Aptsiauri N: 'Hard' and 'soft' lesions underlying the HLA class I alterations in cancer cells: implications for immunotherapy. Int J Cancer 2010;127:249-256.

38 Alegre E, Rizzo R, Bortolotti D, et al: Some basic aspects of HLA-G biology. J Immunol Res 2014;2014:657625.

39 Morandi F, Rizzo R, Fainardi E, Rouas-Freiss N, Pistoia $\mathrm{V}$ : Recent advances in our understanding of HLA-G biology: lessons from a wide spectrum of human diseases. J Immunol Res 2016;2016:4326495.

40 König L, Kasimir-Bauer S, Hoffmann O, et al: The prognostic impact of soluble and vesicular HLA-G and its relationship to circulating tumor cells in neoadjuvant treated breast cancer patients. Hum Immunol 2016;77:791-799.

41 Engels CC, Fontein DB, Kuppen PJ, et al: Immunological subtypes in breast cancer are prognostic for invasive ductal but not for invasive lobular breast carcinoma. Br J Cancer 2014;111:532-538.

42 De Kruijf EM, Engels CC, van de Water W, et al: Tumor immune subtypes distinguish tumor subclasses with clinical implications in breast cancer patients. Breast Cancer Res Treat 2013;142:355-364.

43 Jeong S, Park S, Park BW, et al: Human leukocyte antigen-G (HLA-G) polymorphism and expression in breast cancer patients. PLoS One 2014;9:e98284.

44 Urosevic M, Dummer R: Human leukocyte antigen-G and cancer immunoediting. Cancer Res 2008;68:627630.

45 Haghi M, Hosseinpour Feizi MA, Sadeghizadeh M, Lotfi AS: 14-bp Insertion/deletion polymorphism of the HLA-G gene in breast cancer among women from North Western Iran. Asian Pac J Cancer Prev 2015; 16: 6155-6158.

46 Madjd Z, Spendlove I, Moss R, et al: Upregulation of MICA on high-grade invasive operable breast carcinoma. Cancer Immun 2007;7:17.
47 Ishibashi K, Kumai T, Ohkuri T, et al: Epigenetic modification augments the immunogenicity of human leukocyte antigen $\mathrm{G}$ serving as a tumor antigen for T cellbased immunotherapy. Oncoimmunology 2016;5: e1169356.

48 Dong H, Strome SE, Salomao DR, et al: Tumor-associated B7-H1 promotes T-cell apoptosis: a potential mechanism of immune evasion. Nat Med 2002;8:793800.

49 Martin-Villalba A, Llorens-Bobadilla E, Wollny D: CD95 in cancer: tool or target? Trends Mol Med 2013; 19:329-335.

50 Bergmann-Leitner ES, Abrams SI: Positive and negative consequences of soluble Fas ligand produced by an antigen-specific CD4(+) $\mathrm{T}$ cell response in human carcinoma immune interactions. Cell Immunol 2001;209: 49-62.

51 Gutierrez LS, Eliza M, Niven-Fairchild T, Naftolin F, Mor G: The Fas/Fas-ligand system: a mechanism for immune evasion in human breast carcinomas. Breast Cancer Res Treat 1999;54:245-253.

52 Le Gallo M, Poissonnier A, Blanco P, Legembre P: CD95/Fas, non-apoptotic signaling pathways, and kinases. Front Immunol 2017;8:1216.

53 Fulda S, Meyer E, Debatin KM: Inhibition of TRAILinduced apoptosis by Bcl-2 overexpression. Oncogene 2002;21:2283-2294.

54 Zhivotovsky B, Orrenius S: Carcinogenesis and apoptosis: paradigms and paradoxes. Carcinogenesis 2006 27:1939-1945.

55 Ryan BM, Konecny GE, Kahlert S, et al: Survivin expression in breast cancer predicts clinical outcome and is associated with HER2, VEGF, urokinase plasminogen activator and PAI-1. Ann Oncol 2006;17:597-604

56 Devarajan E, Sahin AA, Chen JS, et al: Down-regulation of caspase 3 in breast cancer: a possible mechanism for chemoresistance. Oncogene 2002;21:8843-8851.

57 Grigoriev MY, Pozharissky KM, Hanson KP, Imyanitov EN, Zhivotovsky B: Expression of caspase-3 and -7 does not correlate with the extent of apoptosis in primary breast carcinomas. Cell Cycle 2002;1:337-342.

58 Alsaab HO, Sau S, Alzhrani R, et al: PD-1 and PD-L1 checkpoint signaling inhibition for cancer immunotherapy: mechanism, combinations, and clinical outcome. Front Pharmacol 2017;8:561.

59 Taube JM, Galon J, Sholl LM, et al: Implications of the tumor immune microenvironment for staging and therapeutics. Mod Pathol 2017;DOI: 10.1038/ modpathol.2017.156.

60 Jadus MR, Natividad J, Mai A, et al: Lung cancer: a classic example of tumor escape and progression while providing opportunities for immunological intervention. Clin Dev Immunol 2012;2012:160724.

61 Wang X, Sun Q, Liu Q, et al: CTC immune escape mediated by PD-L1. Med Hypotheses 2016;93:138-139.

$62 \mathrm{Li}$ Z, Dong P, Ren M, et al: PD-L1 expression is associated with tumor FOXP3(+) regulatory T-cell infiltration of breast cancer and poor prognosis of patient. J Cancer 2016;7:784-793.

$63 \mathrm{Li} \mathrm{CW}$, Lim SO, Xia W, et al: Glycosylation and stabilization of programmed death ligand-1 suppresses T-cell activity. Nat Commun 2016;7:12632.

64 Bertucci F, Finetti P, Colpaert C, et al: PDL1 expression in inflammatory breast cancer is frequent and predicts for the pathological response to chemotherapy. Oncotarget 2015;6:13506-13519.

65 Bertucci F, Finetti P, Birnbaum D, Mamessier E: The PD1/PDL1 axis, a promising therapeutic target in aggressive breast cancers. Oncoimmunology 2015;5: e1085148.

66 McCullar B, Pandey M, Yaghmour G, et al: Genomic landscape of small cell carcinoma of the breast contrasted to small cell carcinoma of the lung. Breast Cancer Res Treat 2016;158:195-202. 
67 Beckers RK, Selinger CI, Vilain R, et al: Programmed death ligand 1 expression in triple-negative breast cancer is associated with tumour-infiltrating lymphocytes and improved outcome. Histopathology 2016;69:25-34

68 Soliman H, Khalil F, Antonia S: PD-L1 expression is increased in a subset of basal type breast cancer cells. PLoS One 2014;9:e88557.

69 Guo L, Li W, Zhu X, et al: PD-L1 expression and CD274 gene alteration in triple-negative breast cancer: implication for prognostic biomarker. Springerplus 2016;5:805.

70 Uhercik M, Sanders AJ, Owen S, et al: Clinical significance of PD1 and PDL1 in human breast cancer. Anticancer Res 2017;37:4249-4254.

71 Sabatier R, Finetti P, Mamessier E, et al: Prognostic and predictive value of PDL1 expression in breast cancer. Oncotarget 2015;6:5449-5464.

72 Nanda R, Chow LQ, Dees EC, et al: Pembrolizumab in patients with advanced triple-negative breast cancer: phase Ib KEYNOTE-012 study. J Clin Oncol 2016;34: 2460-2467.

73 Denkert C, von Minckwitz G, Brase JC, et al: Tumorinfiltrating lymphocytes and response to neoadjuvant chemotherapy with or without carboplatin in human epidermal growth factor receptor 2-positive and triplenegative primary breast cancers. J Clin Oncol 2015;33: 983-991.

74 Burugu S, Gao D, Leung S, Chia SK, Nielsen TO: LAG$3+$ tumor infiltrating lymphocytes in breast cancer: clinical correlates and association with PD-1/PD-L1+ tumors. Ann Oncol 2017;28:2977-2984.

75 Zhang H, Xiang R, Wu B, Li J, Luo G: T-cell immunoglobulin mucin-3 expression in invasive ductal breast carcinoma: clinicopathological correlations and association with tumor infiltration by cytotoxic lymphocytes. Mol Clin Oncol 2017;7:557-563.
76 Liu C, Liu J, Wang J, et al: B7-H3 expression in ductal and lobular breast cancer and its association with IL10. Mol Med Rep 2013;7:134-138.

77 Petitprez F, Vano YA, Becht E, et al: Transcriptomic analysis of the tumor microenvironment to guide prognosis and immunotherapies. Cancer Immunol Immunother 2017;DOI: 10.1007/s00262-017-2058-z.

78 Fridman WH, Pagès F, Sautès-Fridman C, Galon J: The immune contexture in human tumours: impact on clinical outcome. Nat Rev Cancer 2012;12:298-306.

79 Domschke C, Schuetz F, Ge Y, et al: Intratumoral cytokines and tumor cell biology determine spontaneous breast cancer-specific immune responses and their correlation to prognosis. Cancer Res 2009;69:84208428.

80 Rody A, Holtrich U, Pusztai L, et al: T-cell metagene predicts a favorable prognosis in estrogen receptornegative and HER2-positive breast cancers. Breast Cancer Res 2009;11:R15.

81 De Melo Gagliato D, Cortes J, Curigliano G, et al: Tumor-infiltrating lymphocytes in breast cancer and implications for clinical practice. Biochim Biophys Acta 2017;1868:527-537.

82 Ibrahim M, Scozzi D, Toth K, et al: Naive CD4+ T cells carrying a TLR2 agonist overcome TGF- $\beta$-mediated tumor immune evasion. J Immunol 2018;200:847-856.

83 Zhu S, Lin J, Qiao G, Wang X, Xu Y: Tim-3 identifies exhausted follicular helper T cells in breast cancer patients. Immunobiology 2016;221:986-993.

84 Gu-Trantien C, Loi S, Garaud S, et al: CD4+ follicular helper $\mathrm{T}$ cell infiltration predicts breast cancer survival. J Clin Invest 2013;123:2873-2892.

85 Schmidt M, Böhm D, von Törne C, et al: The humoral immune system has a key prognostic impact in nodenegative breast cancer. Cancer Res 2008;68:5405-5413.
86 Perou C, Jeffrey SS, van de Rijn M, et al: Distinctive gene expression patterns in human mammary epithelial cells and breast cancers. Proc Natl Acad Sci U S A 1999;96:9212-9217.

87 Huang E, Cheng SH, Dressman H, et al: Gene expression predictors of breast cancer outcomes. Lancet 2003;361:1590-1596.

88 Gil Del Alcazar CR, Huh SJ, Ekram MB, et al: Immune escape in breast cancer during in situ to invasive carcinoma transition. Cancer Discov 2017;7:1098-1115.

89 Denkert C, Loibl S, Noske A, et al: Tumor-associated lymphocytes as an independent predictor of response to neoadjuvant chemotherapy in breast cancer. J Clin Oncol 2010;28:105-113.

90 Loi S, Sirtaine N, Piette F, et al: Prognostic and predictive value of tumor-infiltrating lymphocytes in a phase III randomized adjuvant breast cancer trial in nodepositive breast cancer comparing the addition of docetaxel to doxorubicin with doxorubicin-based chemotherapy: BIG 02-98. J Clin Oncol 2013;31:860-867.

91 Ali HR, Provenzano E, Dawson SJ, et al: Association between CD8+ T-cell infiltration and breast cancer survival in 12,439 patients. Ann Oncol 2014;25:15361543

92 Hugo W, Zaretsky JM, Sun L, et al: Genomic and transcriptomic features of response to anti-PD-1 therapy in metastatic melanoma. Cell 2016;165:35-44.

93 Song C, Piva M, Sun L, et al: Recurrent tumor cell-intrinsic and -extrinsic alterations during MAPKi-induced melanoma regression and early adaptation. Cancer Discov 2017;7:1248-1265.

94 Lu L, Xu X, Zhang B, Zhang R, Ji H, Wang X: Combined PD-1 blockade and GITR triggering induce a potent antitumor immunity in murine cancer models and synergizes with chemotherapeutic drugs. J Transl Med 2014;12:36. 